\title{
Hubble, Chandra and Keck Constraints on Massive Galaxy Clusters at $\mathrm{z}=0.2$ and $\mathrm{z}=0.5$
}

\author{
Graham P. Smith ${ }^{1}$ \\ ${ }^{1}$ California Institute of Technology, Department of Astronomy, Mail Code 105-24, Pasadena, \\ CA 91125, USA. email: gps@astro.caltech.edu
}

\begin{abstract}
I present recent observations from two Hubble Space Telescope(HST)/ACS programs that target the most $\mathrm{X}$-ray luminous and thus (presumably) most massive galaxy clusters at $z=0.5$ - the highest redshift at which complete, well-defined samples of such rare systems are available. The first program (GO:9836, PI: R.S. Ellis) exploits a huge mosaic of 41 ACS pointings spanning a $10 \mathrm{Mpc}$ region centered on MS0451-03. This is the largest contiguous space-based image of a cluster to date. I describe a preliminary weak-lensing analysis and a new Keck/DEIMOS redshift catalog of 1000 galaxies in this field. The second program (GO:9722, PI: H. Ebeling) studies the core regions of the twelve most luminous clusters at $z \geqslant 0.5$ from the MAssive Cluster Survey (MACS; Ebeling et al. 2001). Multi-color ACS observations in combination with recent Keck/LRIS spectroscopy of gravitational arcs constrain the distribution of mass in the cluster cores, thus laying the foundation for detailed multi-diagnostic (lensing, $\mathrm{X}$-ray, near-infrared, SZE) investigation of this sample. For example, it is of particular interest to explore how the structure and state of relaxation of massive clusters evolved between this sample at $z \geqslant 0.5$ that measured by Smith et al. (2004, astro-ph/0403588) at $z=0.2$.
\end{abstract}

\section{Introduction}

Massive galaxy clusters observed in the local universe descend from rare high-sigma fluctuations in the primordial power spectrum. Evolution of the high-mass tail of the cluster mass function with look-back-time can therefore tell us about when the mass content (dark matter, hot gas and galaxies) of clusters assembled, and may help to constrain whether the initial matter fluctuations obey Gaussian statistics. Detailed study of the assembly and relaxation history of each matter component will also place constraints on the inter-play between dark and luminous matter, and thus help to constrain the physical properties of dark matter and pin-point its role in the evolution of clusters.

In this proceedings I summarize recently published results and new preliminary analyses from two large ongoing programs that take complementary approaches to untangling the formation and evolution of massive clusters. In $\S 2$ I describe a multi-diagnostic study of the cores of massive clusters in two redshift slices: $z=0.2$ and $z=0.5$, the corner-stone of which is deep HST imaging. Then in $\S 3 \mathrm{I}$ present studies of $\mathrm{Cl} 0024(z=0.4)$ and MS $0451(z=0.54)$ that exploit panoramic $H S T$ imaging out to the turnaround radii $(\sim 5 \mathrm{Mpc})$.

\section{Mapping the Evolution of Massive Clusters}

We are conducting a long-term multi-wavelength program to map the assembly history and thermodynamics of massive clusters in a series of narrow redshift slices, ultimately out to $z>1$. The key to our survey is to combine multiple complementary observational and analytical techniques: gravitational lensing studies of the total mass (e.g. Kneib et al. 1996; Smith et al. 2001); spectroscopic and photometric surveys of cluster galaxies 
(e.g. Czoske et al. 2001, 2002; Kodama et al. 2001); and X-ray spectro-imaging of the hot intracluster gas (e.g. Smith et al. 2004a - S04).

\subsection{Results at $z=0.2$}

We have recently completed the first statistical analysis of the distribution of dark and luminous matter in a homogeneous sample of $10 \mathrm{X}$-ray luminous, and thus massive clusters at $z=0.2$. The overall survey combines deep HST/WFPC2 imaging of the cluster cores (Smith et al. 2001; S04), with panoramic weak lensing observations from CFHT (Czoske 2002; Bardeau et al. 2004) and X-ray observations from both Chandra (S04) and XMM-Newton (Marty et al. 2004). The results described below concentrate on the $H S T$ and Chandra results recently presented by S04.

The HST imaging reveals gravitationally-lensed background galaxies in all 10 clusters (e.g. Fig. 1). This signal is interpreted with a sophisticated ray-tracing code to construct accurate models of the relative mass distribution in the central $1 \mathrm{Mpc}$ of the clusters (Kneib 1993; Smith 2002). Spectroscopic redshifts for the lensed features obtained with Keck (Smith et al. 2001, 2002, S04; Fig. 1) allowed us to constrain the total mass within a cylinder through the cluster core and hence precisely $(<5 \%)$ calibrate our mass models. This absolute calibration is essential to measure the mass of the clusters on small scales (i.e. within the $H S T$ field of view), and on larger scales by normalizing the weak lensing signal (e.g. Kneib et al. 2003).

When combined with analysis of the X-ray spectro-imaging data available from Chan$d r a$ we find that $70 \%$ of these clusters host a dynamically immature core. They therefore appear to have experienced in-fall from the field in the preceding 2-4Gyr and/or are still in the process of relaxing following in-fall at even earlier times (Fig. 1 \& S04). The normalization of the mass-temperature $(\mathrm{M}-\mathrm{T})$ relation for these immature clusters is $40 \%$ hotter than for the relaxed clusters (Fig. 1). A plausible interpretation of this result, suggested by recent simulations (Ricker et al. 2001) is that cluster-cluster merger activity systematically boosts the temperature of the dynamically immature clusters.

If confirmed, this interpretation has far-reaching implications for the use of clusters to measure cosmological parameters such as $\sigma_{8}$ (e.g. Smith et al. 2003). Such experiments rely on converting an observable such as cluster temperature to the cluster mass (e.g. Nevalainen et al. 2000). Of most significance in the coming years are X-ray and SunyaevZeldovich Effect (SZE - e.g. Carlstrom, Holder \& Reese 2002) surveys, which aim to detect large numbers of clusters out to $z \simeq 1$ and then rely on the cluster $\mathrm{M}-\mathrm{T}$ relation to derive constraints on the cosmological equation-of-state parameter $w$. Therefore, unless the $\mathrm{M}-\mathrm{T}$ relation is well-calibrated at both low and high redshift, these experiments will be undermined by poorly calibrated astrophysical systematics.

\subsection{Massive Galaxy Clusters at $z=0.55$}

The next step is to track the evolution of clusters further back in time. We have obtained 42 orbits with $H S T$ / ACS to survey to an identically selected sample of $12 \mathrm{X}$-ray luminous clusters $\left(L_{X} \geqslant 8 \times 10^{44} \mathrm{ergs}^{-1}[0.1-2.4 \mathrm{keV}]\right)$ at $z=0.55 \pm 0.05$ - i.e. $3 \mathrm{Gyr}$ (approximately one cluster-crossing time) prior to $z=0.2$. This is a statistically complete subset of the Xray flux-limited MACS cluster sample (Ebeling et al. 2001). Our deep ACS observations ( 2 orbits in each of $\mathrm{F} 555 \mathrm{~W}$ and $\mathrm{F} 814 \mathrm{~W}$ ) provide $\mathrm{FWHM}=0.1^{\prime \prime}$ imaging of lensed features on scales from $10 \mathrm{kpc}$ to $1.5 \mathrm{Mpc}$ and hence constrain the total relative mass distribution on these scales. We typically identify 3-10 multiple-image candidates per cluster (see Fig. 2 for an example, including recent Keck spectroscopy of a multiple-image system at $z=1.89)$. 

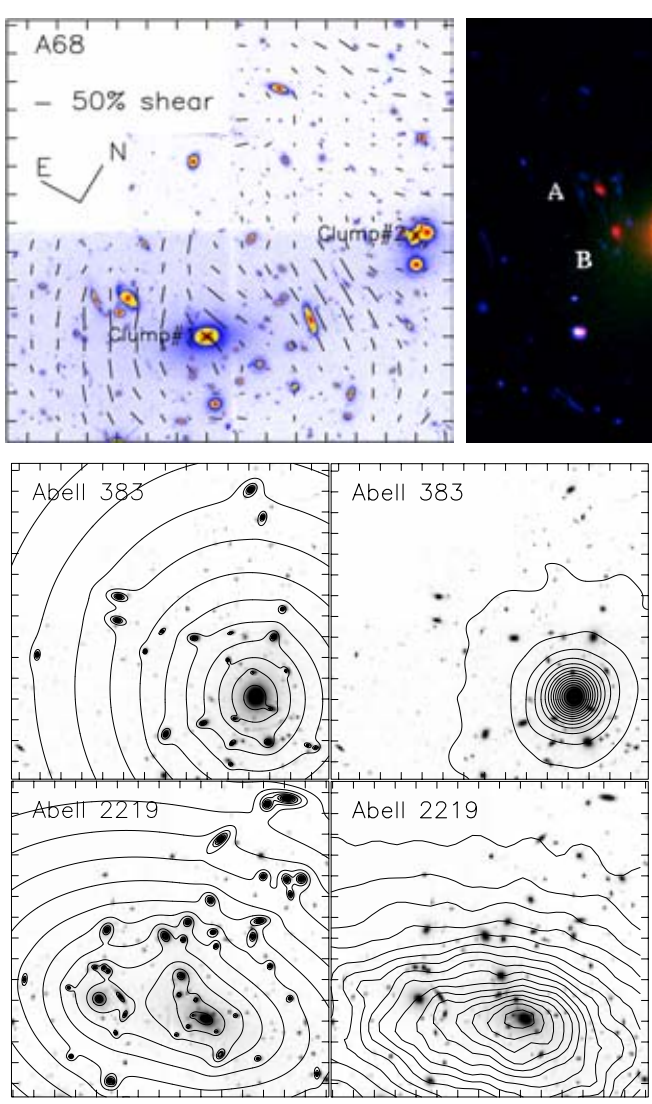
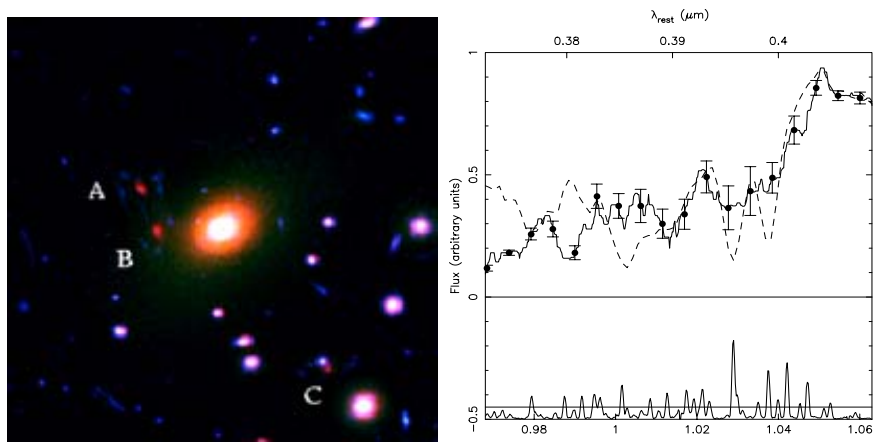

$\lambda_{\text {obs }}(\mu m)$

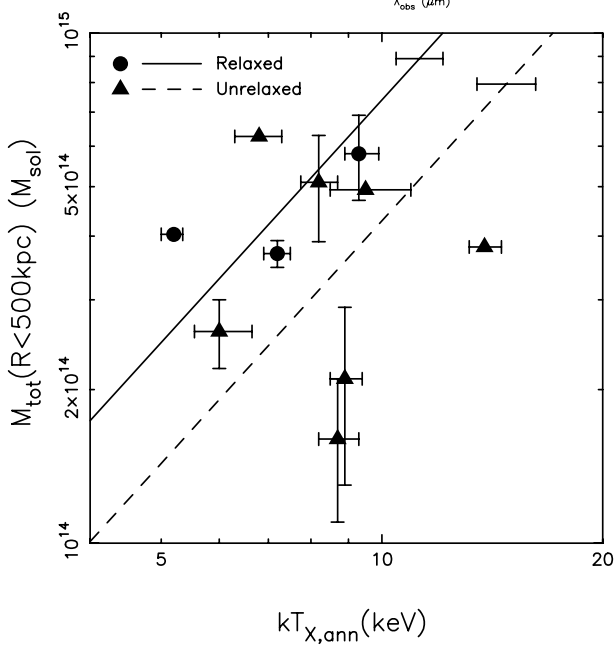

Figure 1. UPPER - Example data from the experiment at $z=0.2$ described in $\S 2.1$. To the left is shown the WFPC2 data for A $68(z=0.25)$ with vectors overplotted to show the amplitude of the weak shear signal. In the middle, a zoom ( $R K$-bands) into the central $50^{\prime \prime} \times 50^{\prime \prime}$ region shows a triply-imaged extremely red galaxy (images A,B,C) for which we secured a redshift of $z=1.6$ with NIRSPEC on Keck (See right most panel - Smith et al. 2002a, 2002b). Spectroscopic redshifts are essential for constructing detailed models of the mass distribution in the cluster cores. LOWER LEFT - The mass map for A 383 (top left) and A 2219 (lower left), as examples of relaxed (3 of the 10) and unrelaxed (7) clusters respectively. The right hand panels show the $\mathrm{X}$-ray flux contours from Chandra for these two clusters. LOWER RIGHT - Mass-temperature plane for the ten clusters at $z=0.2$, showing the hotter normalization of this relation for unrelaxed clusters, as compared with relaxed clusters - see S04 and Smith et al. 2003 for more details.

We also have high-resolution Chandra X-ray spectro-imaging and panoramic subarcsecond resolution $V R z$ Subaru imaging in hand and will repeat the analysis performed at $z=0.2$ (S04). A key deliverable from this project will be a precise calibration of the $\mathrm{M}-\mathrm{T}$ relation at $z=0.55$ (the highest redshift at which a well-defined sample of massive clusters is available - Ebeling et al. 2001), and will test for evolution in the structural segregation of clusters in this relation.

\section{Panoramic Weak-lensing Studies of Clusters at $\mathrm{z} \simeq 0.5$}

I now turn to a program that aims to combine the high-fidelity weak- and stronglensing signals measurable with $H S T$ with detailed studies of cluster galaxies. The main aims are to explore the large-scale structure of clusters and the relationship between the cluster environment and cluster galaxy evolution - out to projected physical scales of $5 \mathrm{Mpc}$ from the cluster center. This proceeding concentrates primarily on the lensing 

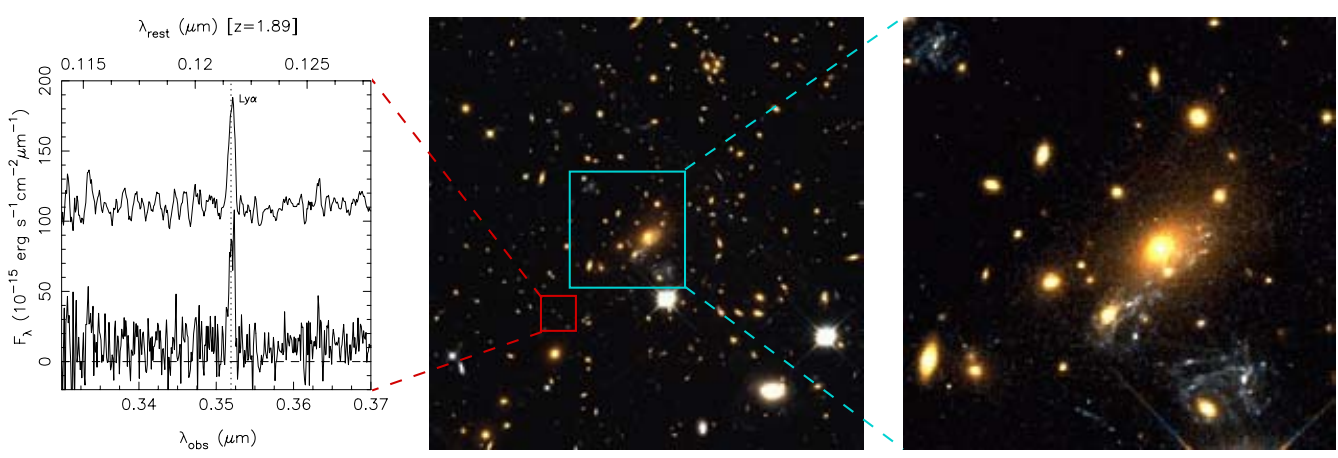

Figure 2. An example from the MACS ACS sample described in $\S 2.2$. In the central panel we show the core of MACS J1149, based on the $V_{606}$ and $I_{814}$ ACS imaging. Zooming right into the brightest central galaxy ( $\mathrm{BCG}$ - right panel), we see a triply-imaged disk galaxy, with candidate radial counter images also marked. We have already obtained a redshift for a faint image pair below left of the BCG using LRIS on Keck-I (Smith et al. in prep.) - see left panel for the spectrum. The unrivaled blue sensitivity of LRIS was vital to achieve this measurement - the identification a single line blueward of the rest wavelength of [OII] leading to a secure redshift determination.

aspects of this project, and readers are referred to Treu et al. (2003), Smith et al. (2004b) and Moran et al. (2004, in prep.) for a full description of the the galaxy evolution component.

\subsection{Results from $\mathrm{Cl} 0024$}

I begin with a comprehensive lensing analysis of the rich cluster $\mathrm{Cl} 0024+1654(\mathrm{z}=0.395)$ based on panoramic sparse-sampled imaging conducted with the WFPC2 and STIS cameras on board the Hubble Space Telescope. By comparing higher fidelity signals in the limited STIS data with the wider field data available from WFPC2, Kneib et al. (2003) demonstrate the ability to detect reliably weak-lensing signals to a cluster radius of $5 \mathrm{Mpc}$, where the mean shear is $\sim 1 \%$. This enabled the study of the distribution of dark matter with respect to the cluster light over an unprecedented range of cluster radii and environments. The projected mass distribution reveals a secondary concentration representing $30 \%$ of the overall cluster mass, which is also visible in the distribution of cluster member galaxies.

Kneib et al. derived the projected mass profile of the main cluster taking into account the influence of the secondary clump. The mass profile determined from the shear was normalized by assuming that background galaxies selected with $23<I<26$ have a redshift distribution statistically similar to that inferred photometrically in the Hubble Deep Fields. The total mass within the central region of the cluster was then independently determined from strong-lensing constraints according to a detailed model that utilizes the multiply imaged arc at $z=1.675$. Combining strong and weak constraints, the mass profile of the cluster was thus probed on scales of $0.1-5 \mathrm{Mpc}$, providing a valuable test of the universal form proposed by Navarro, Frenk \& White (1997 - NFW) on large scales. A generalized power-law fit indicates an asymptotic three-dimensional density distribution of $\rho \propto r^{-n}$ with $n>2.4$. An isothermal mass profile is therefore strongly rejected, whereas an NFW profile with $M_{200}=6.1_{-1.1}^{+1.2} \times 10^{14} h_{65}^{-1} M_{\odot}$ provides a good fit to the lensing data.

This the first time that an observational study has identified a statistically significant difference between the goodness of fit of an NFW and isothermal model. The huge fieldof-view (i.e. dynamic range in the radial direction), superb image quality from $H S T$ and combined strong/weak-lensing constraints were all vital to this achievement. 

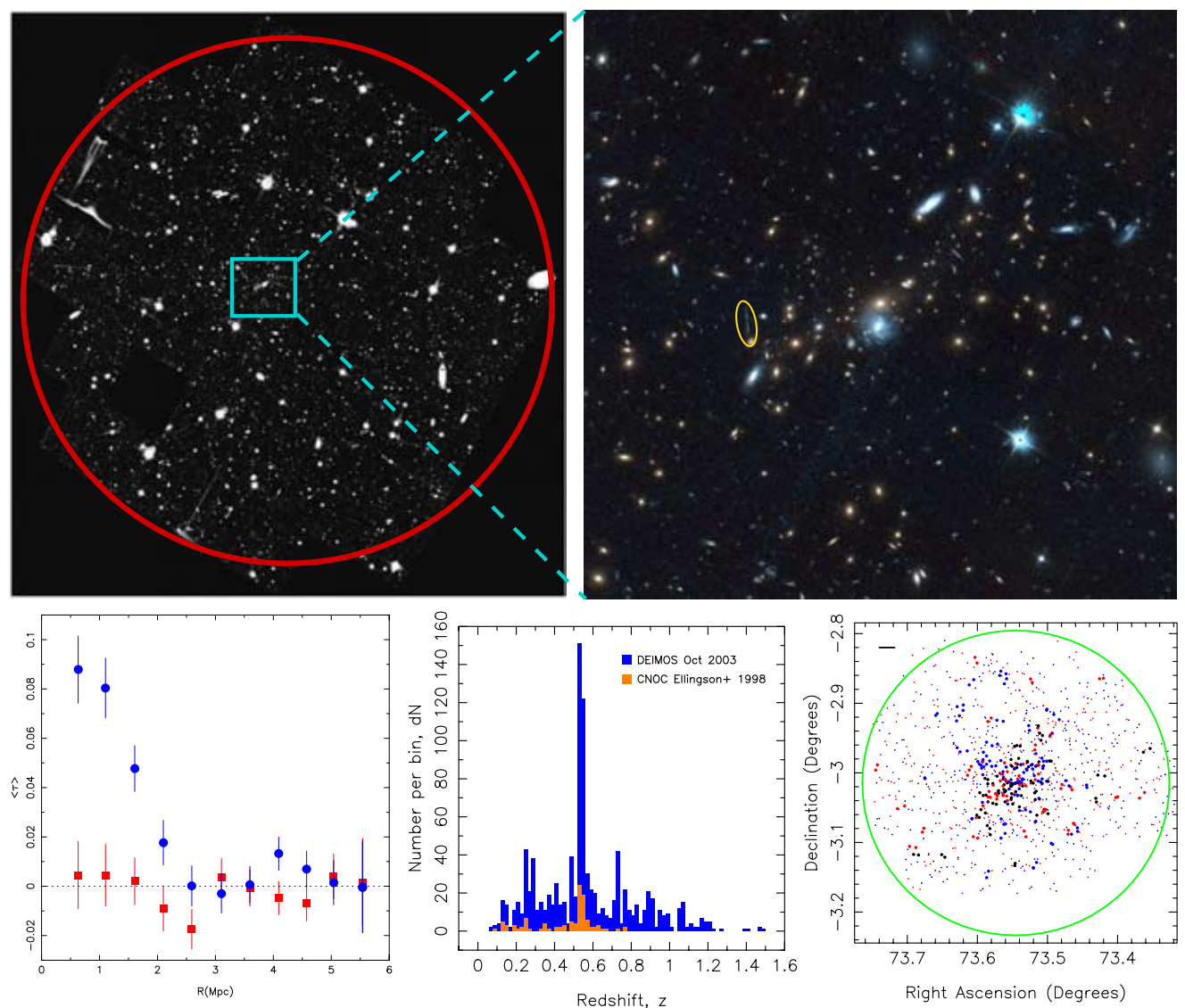

Figure 3. UPPER LEFT - The full, contiguous ACS mosaic of MS 0451, consisting of 41 pointings. The red circle shows a region of radius $5 \mathrm{Mpc}$ - the scale probed by the sparse WFPC2 mosaic of $\mathrm{Cl} 0024$. UPPER RIGHT - The central $\sim 4 \mathrm{arcmin}^{2}$ of the mosaic. The orange galaxies are cluster members, and the blue galaxies, including the one immediately below the brightest cluster galaxy belong to a foreground group. The arc marked by the gold ellipse lies at $z=2.9$ (Borys et al. 2004) and will be crucial in our combined strong/weak-lensing analysis of this cluster. LOWER LEFT - Preliminary weak shear profile derived from the ACS data, and without any PSF correction to date. The blue data points are the tangential shear measurements in each radial bin, and the red points are the $45^{\circ}$ test. The non-zero red points reveal systematics (probably due to not removing the ACS PSF) that are not yet under control. However the clear detection of a $\sim 1 \%$ signal at $4 \mathrm{Mpc}$ without making any effort to correct the PSF is very encouraging for our more careful analysis that will attempt to map the 2D infall around this cluster. LOWER CENTER - Redshift histogram from our new DEIMOS survey at Keck. Cross-correlation between our SD lensing analysis and the angular/redshift distribution of galaxies will be key to mapping the infall onto the cluster. LOWER RIGHT - We illustrate the power of the redshift catalog by plotting as heavy points galaxies with a neighbor within $R \leqslant 400 \mathrm{kpc}$ and a redshift within $1000 \mathrm{~km} / \mathrm{s}$. Blue galaxies lie in front of the cluster, black in the cluster and red behind the cluster. Many galaxy groups are clearly present in the surrounding field. the green circle marks a radius of $5 \mathrm{Mpc}$.

\subsection{Preliminary Analysis of MS 0451}

The field-of-view and sensitivity gain of the Advanced Camera for Surveys (ACS) over WFPC2 offers important new opportunities in weak-lensing studies of clusters. In a new study, we have acquired a contiguous mosaic of 41 ACS pointings centered on the $\mathrm{X}$-ray luminous cluster MS 0451-03 at $\mathrm{z}=0.54$ (Fig. 3). This was achieved with half 
the observing allocation required for the sparse-sampled WFPC2 mosaic of $\mathrm{Cl} 0024$. These new data will therefore enable us to test whether the steep outer slope of the dark matter density profile of $\mathrm{Cl} 0024$ is representative of other clusters. The complete angular coverage across a region of $10 \mathrm{Mpc}$ will also allow a detailed investigation of infall onto this cluster from the surrounding field. As such it will therefore be an ideal complement to the MACS study of the cluster cores described in $\S 2.2$.

A preliminary weak shear profile is presented in Fig. 3. This is based on galaxy shape measurements using SExtractor without any correction for distortions in the pointspread-function (psf). Therefore unsurprisingly, there is evidence for systematic errors in the data that have yet to be removed (see the radial range $2-3 \mathrm{Mpc}$ ). However the detection of a $1 \%$ shear signal at $4 \mathrm{Mpc}$ radius, and convergence of the tangential shear to zero at $5 \mathrm{Mpc}$ both bode very well for improving upon the precision of the results derived from $\mathrm{Cl}$ 0024, both in one- and two-dimensions. Indeed, numerous talks at this meeting (e.g. Rhodes et al., Heymans et al., Albert et al.) all confirm that the task of characterizing the ACS psf is not yet complete.

To illustrate the potential of these data to probe the in-fall of matter onto MS 0451, I show in Fig. 3 the redshift distribution from our new (huge) redshift catalog in this field, recently compiled from Keck/DEIMOS observations by Moran et al. (2004, in prep.). The redshift histogram (1000 galaxies) and two-dimensional distribution of galaxies on the sky demonstrate that cross-correlating the mass map derived from the lensing analysis with the redshift catalog should be a powerful technique to probe in-fall in three-dimensions.

\section{Acknowledgements}

I thank my collaborators for allowing me to present preliminary results based on unpublished data: H. Ebeling, A.C. Edge, R.S. Ellis, J.-P. Kneib, S.M. Moran, I. Smail and T. Treu. I also acknowledge receipt of a grant from the IAU in support of my attendance at this meeting.

\section{References}

Bardeau S., et al., 2004, AESA, submitted.

Borys C., et al., 2004, MNRAS, 352, 759-767.

Carlstrom J., et al., 2002, ARA $\mathscr{\mho} A$, 40, 643-680.

Czoske O., 2002, PhD Thesis, Université Paul Sabatier, Toulouse, France.

Czoske O., et al., 2001, A\& $\&$ A, 372, 391-405.

Czoske O., et al., 2002, A\&̈A, 386, 31-41.

Ebeling H., et al., 2001, ApJ, 553, 668-676.

Kneib J.-P., 1993, PhD Thesis, Université Paul Sabatier, Toulouse, France.

Kneib J.-P., et al., 2003, ApJ, 598, 804-817.

Kneib J.-P., et al., 1996, ApJ, 471, 643-656.

Marty P.B., et al., 2004, $A \mathscr{E} A$, submitted.

Navarro J., et al., 1997, ApJ, 490, 493-508.

Ricker P., et al., 2001, ApJ, 561, 621-644.

Smith G.P., et al., 2001, ApJ 552, 493-503.

Smith G.P., 2002, PhD Thesis, University of Durham, UK.

Smith G.P., et al., 2002a, MNRAS 330, 1-16.

Smith G.P., et al., 2002b, MNRAS 333, L16-20.

Smith G.P., et al., 2003, ApJ 590, L79-82.

Smith G.P., et al., 2004a, MNRAS, submitted, astro-ph/0403588.

Smith G.P., et al., 2004b, ApJ, submitted, astro-ph/0403455.

Treu T., et al., 2003, ApJ, 591, 53-78. 